\title{
FONTS BIBLIOGRÀFIQUES I DOCUMENTALS EN ANTROPOLOGIA DE LA MEDICINA I LA SALUT'?
}

Josep Maria Comelles Departament d'Antropologia Cultural, Tarragona Institut Català d'Antropologia 
La introducció en l'àmbit acadèmic de l'Antropologia de la Medicina a casa nostra, està vinculada al desenvolupament de l'Antropologia Cultural i Social a les Facultats de Filosofia i Lletres.

És dins l'especialitat d'Antropologia que han de formar-se els especialistes en aquest camp, malgrat que en algunes facultats, els Departaments d'Història de la Medicina han afavorit orientacions pròximes a la nostra especialitat. Però, com ja ha estat posat de manifest a les 1es. Jornades d'Antropologia de la Medicina que se celebraren el passat desembre del 1982 a Tarragona, el desenvolupament i la formació en Antropologia de la Medicina no poden romandre fora de la formació general de l'antropòleg.

La vinculació de l'Antropologia Social i Cultural a les Facultats de Lletres suposa, però, un problema greu per a la formació d'antropòlegs de la Medicina: la seva separació i l'aïllament respecte al sector sanitari i, en general, a la problemàtica de les Ciències Naturals; no existeix una Història de la ciència a les Facultats... d'Història, i la Història de la Medicina o la Salut Pública no són matèries que els estudiants puguin escollir dins els seus curriculums "literaris".

És per això que el Seminari d'Antropologia de la Medicina del Departament d'Antropologia Cultural de Tarragona es proposa la publicació d'una sèrie d'articles, destinats especificament als estudiants de lletres, que els permetin disposar d'una documentació de base indispensable per a moure's dins el camp de la salut i la malaltia a la 
nostra societat. Aquest primer lliurament té com a objecte donar pistes respecte a les fonts bibliográfiques en general. En un proper número d'ARXIU publicarem una bibliografia bàsica en Antropologia de la Medicina, i els membres del seminari procuraran editar recensions de textos d'actualitat que puguin tenir interès per als antropòlegs, des d'aquesta perspectiva.

Aquest repertori fou publicat per primera vegada el 1977 a la Revista ETHNICA de Barcelona. Els sis anys que han passat I'havien convertit en gairebé inútil, especialment en allò que fa referència a la Peninsula Ibèrica i a Catalunya, en particular. Érem, aleshores, al principi d'un desenvolupament que ara, sortosament, permet disposar d'un fons bibliogràfic força important.

(") El meu agraïment a Xavier Granero per la seva col.laboració en el capitol de revistes.

\section{BIBLIOGRAFIA INTERNACIONAL.}

1.1. Reculls bibliogràfics internacionals que publiquen material sobre Antropologia de la Medicina i Ciències de la Salut.

1. CLAUSEN. J.A.

1956 Sociology and the field of Mental Health. New York, Russell Sage. 96 referències sobre aspectes sòcio-culturals de la malaltia mental. És el primer repertori disponible.

2 - FREEMANN, H.E. y REEDER, L.G.

1957 Medical Sociology: a review of literature. Amer. Sociol. Rev., 22(1) 73-81.

Revisió de 20 col.leccions de revistes i actes de congressos: 107 referències.

3. FREIDSON, E.

1961-62 The Sociology of Medicine. A trend report and bibliography. Current Sociology 10-11 (3) 123-192.

4. HARVARD MEDICAL SCHOOL

1962 Community Mental Health and Social Psychiatry. Harvard University Press.

5. SIMMONS, O.G.

1963 "Social Research in Health and Medicine: A Bibliography A: Freemann, H.E., Levine, S. y Reeder, L.G.

Handbook of Medical Sociology.

Englewood Cliffs. Prentice Hall, pp: 483-581

És la primera edició del que ressenyem al $n^{\circ} 10$.

6. PEARSALL, $M$.

1963 Medical Behavioral Sciences: a Selected Bibliography. Lexington University Press.
7 - DRIVER, E.G.

1965 The Sociology and Anthropology of Mental IIIness. Amherst: University of Massachussets Press.

És el primer gran repertori especialitzat en aquestes temàtiques. Conté unes 1500 referències entre els anys 1956 i 1963. Continua la tasca de 1.

8 - DUFRANCATEL, C.

1968 La sociologie des maladies mentales. Current Sociology. La sociologie contemporaine, XVI (2)Paris - La Haya, Mouton.

Revisió molt complerta del tema fins al 1968. Bibliografia no exhaustiva però comentada (unes 800 referències). És el repertori fonamental per a començar a treballar en aquest camp.

9. MECHANIC, D.

1968 Medical Sociology. New York: Russell Sage pp: 455-484.

10 - FREEMANN, H.E., LEVINE, S. y REEDER, L.G.

1972 Handbook of Medical Sociology. Englewood Cliffs: Prentice Hall. És la segona edició $\left(g . n^{\circ}\right.$ ) d'un manual clàssic americà. Gairebé tots els articles contenen bibliografies molt extenses sobre la ma. for part dels temes clássics que fan referència a la Sociologia de la Medicina i als estudis institucionals. El seu límit cronològic és el 1970. Conté tres articles molt importants des del punt de vista bibliogràfic:

SIMMONS, O.G. Y BERKANOVIC, E.

1972 "Social Research in Mental Health and Medicine: a Bibliography", pp: 523-584.

CLAUSEN, J.A.

1972 "The sociology of mental disorder", pp: 168-188: Conté 76 referències bibliogràfiques. És una actualització del 1.

GROG, S.H. y VER STEEG, D.F.

1972 "The hospital as a social system", pp: 274-314. 279 referències bibliogràfiques. És un article fonamental per al camp concret dels estudis sobre organització social d'hospitals; les referències són gairebé exclusivament americanes.

\section{1 - DRIVER, E.D.}

1972 The Sociology and Anthropology of Mental IIIness: a Reference guide. Ahmerst: Univ. of Massachussets Press.

guide. Anmerst: $n$ niv. tada) fins al 1968: 5910 referències. Hi ha un esforç notable per recollir bibliografia no nord-americana.

12. STEUDLER, François.

1972 "Le champ de la sociologie médicalen, a: Sociologie Médicale. Pa-

ris, Armand Colin.
Excel.lent introducció a la Sociologia de la Medicina, seguida 
d'un repertori bibliogràfic àmpliament comentat, amb 123 referències; n'hi ha moltes d'europees, especialment franceses, i una interdisciplinarietat molt ben combinada. És un repertori desitjable per a l'estudiant no iniciat.

13 - MIGUEL, Jesús de.

1975 Apéndice: Bibliografía básica de Sociologia de la Medicina. Pa. pers. (Barcelona) 5: 241-269

Excel.lent recull de bibliografia bàsica en Sociologia de la Medicina: 89 referències dels grans textos bàsics americans, en aquest terreny. Lamentablement, hi ha un buit important de bibliografia europea. Es complementa molt bé amb Steudler (1972) i Dufrancatel (1968).

14 - COOPER, B.B. i SHEPHERD, M

1973 "Epidemiology and Abnormal Psychology». A: Eysenck, Handbook of Abnormal Psychology: Londres, Pitman.

Revisió molt complerta fins al 1969, en el terreny de l'epidemiolo. gia psiquiàtrica. Predomini d'estudis quantitatius.

15 - COOPER. B.G. I MORGAN, H.G.

1973 Epidemiological Psychiatry.

Springfield, III. Charles C. Thomas (Trd. cast. PANAP, 1974, edició exhaurida).

16- ALLUÉ, Marta.

1983 Mort i Ciències Sociais: una bibliografia:

Arxiu d'Etnografia de Catalunya, 2.

Més de 390 referències sobre el tema de la mort i l'agonia, en Antropologia, arreu del món. Incidència especial en el tema de la mort en l'àmbit sanitari.

17 - RÉVUE FRANÇAISE DE SOCIOLOGIE.

1973 Guide sociologique en sociologie médicale. Publications en lan. gue française. Revue Française de Sociologie XIV 137.193 Número especial dedicat a la sociologia de la medicina.

18 - LE CERF, J.F. Y SEBILLE, G

1975 Dimensions sociales de la psychopatologie et de la psychiatrie. Bibliographie en langue française. Rev. Française de Sociologie XVI, 3, 380-417.

Malgrat que amb aquestes publicacions pot iniciar-se, en línies generals, una recerca aprofundida, bibliogràfica i documental, excepció feta del número 16 , la resta de repertoris no donen dades més que fins el 1970.

\subsection{REPERTORIS INTERNACIONALS DE BIBLIOGRAFIA.}

Per a una investigació de les fonts més próximes en el temps cal recórrer als indexs bibliogràfics internacionals, que són molt poc còmodes d'utilitzar -en aquest camp- a excepció dels que citarem seguidament. D'altra banda, alguns són difícilment accessibles a casa nostra.

\section{Index to American Doctoral Dissertations}

\section{0 - Dissertation Abstracts}

Són publicacions de resums de tesis de doctorat. Cal manejar-los perquè contenen una documentació imponent en aquests camps. La seva consulta té interès per a l'investigador avançat, no tant per al principiant.

1. International Index to Social Sciences: Sociology. Londres, Tavistock.

22 - International Index to Social Sciences: Anthropology.

Londres, Tavistock.

Són llibres d'aparició anual que recullen bibliografia no comentada. Són força exhaustius i manejables. El retard que duen en relació a la publicació original oscil.la entre l'any i l'any i mig. Bona quantitat de revistes no anglo-saxones ressenyades. Disponibles quantioteca del Departament d'Antropologia Cultural de Barcea la Biblioteca del Departament d'Antropologia Cultural (Barcelona) i Biblioteca Universitària de Tarragona.

23. Bulletin Signaléctique de CNRS: Sociologie/Ethnologie. Paris, Maison des Sciences de l'Homme.

Butlleti trimestral, particularment útil des del 1972, en què va ésBur intro informàtic (PASCAL). Hom pot sol.licitarne fotocòpies d'articles o resums a preus no módics però tampoc ne fotocòpies d'articles o resums a preus no d'uns sis mesos, però imposs resums són força extensos, i es molt manejable. Disponible a la Biblioteca del Departament d'Antropologia Cultural, de Barcelona $i$ al Centro de Etnologia Peninsular de Barcelona.

\subsection{Bancs de dades.}

En els darrers temps, tots els països estan organitzant bancs de dades. A casa nostra, comencen a funcionar-ne alguns, i cal pensar que d'aqui a uns casa noster elements bàsics per a aquest tipus de treball. Els lectors hauran, però, d'informar-se'n a cada una de les localitats on resideixin.

Centre de Documentació e informática biomédica.

Facultad de Medicina, Universidad de Valencia.

Es un banc de dades, actualment el mes complert que hi ha a to l'estat en aquesta temàtica. Conectat amb algun dels principals l'estat en aquesta temática. circuits d'informació internacional i com a editor del Indice Médi-
co Español, disposa de dades sobre tota la producció biomédica espanyola. 


\section{REVISTES.}

Fora impossible donar una llista complerta de totes aquelles publicacions que fan referència a temes d'interès per a l'antropòleg de la Medicina i la Salut. Les que citem a continuació serveixen únicament per a donar pistes a l'estudiant que comença en aquest terreny. Per això utilitzem el criteri d'ésser relativament exhaustius amb les revistes especialitzades, i només donem algunes pistes que es refereixen a les revistes generals que inclouen, eventualment, articles que atenyen aquest camp. La revisió dels reculls reunits a 1.1 pot donar moltes més col.leccions que les que aci proposem.

\subsection{Revistes especialitzades.}

Journal of Health and Social Behavior (ASA, Chicago).

Revista de Sociologia de la Medicina, publicada per l'Associació Americana de Sociòlegs. Manté una línia força tradicional, amb un predomini d'articles empirics. Nombrosos números monogràfics. La seva consulta és absolutament Indispensable.

Social Science and Medicine. (Pergamon, Londres, Nova York). Una de les més prestigioses, actualment. Publica sis col.leccions diferents (Antropologia, Sociologia, Salut Pública, etc.) i alterna treballs empirics $\mathrm{i}$ teórics des de perspectives sovint força crítiques.

Culture, Medicine and Psychiatry. (D. Reidel, Dordrecht)

Revista dirigida per Arthur Kleinman. Representa els corrents més innovadors en el camp de l'Antropologia de la Medicina, i manté una trajectòria coherent. És indispensable la seva consulta.

Journal of Medicine and Phylosophy. (D. Reidel. Dordrecht).

Dins la línea de l'anterior però des d'una perspectiva molt més interdisciplinària. Ėmfasi en Bioètica.

Theoretical Medicine (abans, Metamedicine) (D.Reidel, Dordrecht) Dins la línea de les dues precedents, revista molt orientada en la direcció de la metodología d'análisi del sector sanitari i de les ciències biomèdiques.

Transcultural Psychiatric Research Review. (Montreal, Mc. Gill University)

Revista quatrimestral d' "abstracts". Conté recensions de caire in terdisciplinari, i articles d'actualització sobre temes concrets. Predomini de la temàtica transcultural.

Ethnopsychiatrica. (Paris, La Pensée Sauvage).
Revista bianual en francès, prioritàriament vinculada al grup de treball de Georges Devereux, que n'és el director.

Medical Anthropology Newsletter. (Society for Medical Anthropology).

Revista editada des de fa una dotzena d'anys, que reflecteix, sobretot, problemes de delimitació del camp, i articles teòrics.

Psychopathologie africaine. (Dakar).

Revista editada a Dakar pel grup del malaguanyat Collomb, i que ha publicat força estudis sobre psiquiatria transcultural.

Medical Anthropology. Cross Cultural Studies in Health and III. ness. (Departament of Anthropology, University of Connecticut). Redgrave, Publishing Co. P.O. Box 67 South Salem, N.Y. 10590

Bulletin de liaison du Séminaire Mensuel d'Ethnomedecine.

(Societé d'Ethnobotanique et d'Ethnozoologie: 7, Rue Cuvier, 7005 Paris).

\subsection{Altres revistes:}

Resulta completament impossible un llistat complet de les revistes que poden ésser útils. Diria que, com a minim, cal sondejar les revistes generals d'Antropologia, Sociologia, Salut Pública, Psiquiatria i História de la Medicina.

\section{FONTS BIBLIOGRÀFIQUES SOBRE CATALUNYA I L'ESTAT ES. PANYOL.}

En la primera edició d'aquest article, fa sis anys, aquest capitol era molt migrat. Posteriorment, s'ha ampliat extraordinàriament, i volem intentar, si no ésser exhaustius, que és força difícil, ésser, almenys, útils.

\subsection{Centres de Documentació en Antropologia de la Medicina.}

No hi ha a Catalunya cap centre especialitzat en aquest camp que disposi de documentació exhaustiva. Proposem, aleshores, una sèrie d'institucions on hom pot obtenir informació o documentació interessant:

Biblioteca de l'Acadèmia de Ciències Mèdiques de Catalunya i Balears. (Passeig de la Bonanova, 47. Barcelona-17)

És una Biblioteca general de Ciències Mèdiques, on hom pot trobar un fons de revistes molt important, amb pràcticament quasi tota la producció catalana i espanyola, i al mateix temps, un fons bibliogràfic suficientment ampli sobre informació general referida al sector sanitari. Accés lliure. Servei de fotocópies. 
- Centro de Documentación en Historia de la Medicina. (Degà Bahi, 59. Barcelona).

Fons bibliogràfic especialitzat en História de la Medicina, i gràcies a l'entusiasme del director d'aquesta biblioteca, Josep Danón, és, probablement, un dels centres més adients per a trobar-hi allò que és impossible d'obtenir en altres llocs. D'altra banda, la catalogació és excel.lent i el seu fitxer permet accedir a dades impensables. Funciona els matins, $i$ hi ha un servei de fotocópia.

- Biblioteca de la Facultat de Medicina de Barcelona (Casanova, 143)

Biblioteca moit important, especiaiment en la seva secció històrica. Moltes col.leccions de revistes espanyoles i estrangeres. Accés lliure, però problemes constants d'espai per a seure. Servei de fotocópies.

Centro de Etnologia Peninsular. (Egipciaques, 9-11. Barcelona). Biblioteca especialitzada en Antropologia. Conté algunes col.lec cions de revistes importants, però un fons bibliogràfic heterogeni. En els darrers temps, accés limitat. Servei de fotocòpies.

- Biblioteca del Departament d'Antropologia Cultural de Barcelo. na. (Fac. Geografia i Història. Zona Universitària).

Biblioteca especialitzada (en formació). Fons bibliogràfic heterogeni, general, i escasses col.leccions de revistes.

Servei de fotocòpies. Acces lliure.

- Biblioteca I Arxiu de l'Hospital de la Sta. Creu i Sant Pau. (Av. Sant Antoni $\mathrm{M}^{\mathrm{a}}$ Claret, 167. Barcelona-25)

Importantissim arxiu històric -en bona part encara inèdit. Fonamental per a seguir l'evolució del món mèdico-sanitari a Catalunya. Biblioteca especialitzada en Medicina; moltes col.leccions de revistes. Accés amb autorització. Servei de fotocòpies.

GSM: Grup de Sociologia de la Medicina. (Apartat 4907. Barcelona).

Reuneix un centenar de sociòlegs i gairebé tots els antropòlegs de la Medicina del nostre país. Vg: Salud y Sociedad.

GAPS. (Passeig de la Bonanova, 47. Barcelona).

Delegacions territorials a Tarragona, Girona i Lleida.

Centre d'Estudis, que edita i encarrega estudis de caire socioló gic en el camp de la salut pública i de l'assistència sanitària. Important fons de revistes i altres publicacions sobre Salut Pública. Accés lliure.

Institut d'Estudis de la Salut. (Avda. Roma, 157. Barcelona) Institució depenent de la Conselleria de Sanitat de la Generalitat, de creació recent (1980). Organitza cursets i promociona estudis en el camp de la salut pública.

Biblioteca del ICESB. (Enric Granados, 2. Barcelona).

Biblioteca especialitzada en Ciències Socials. Fons de revistes important. Acces Iliure. Servei de Fotocopies.

- Seminari d'Antropologia de la Medicina. (Arxiu d'Etnografia de Catalunya. PI. Imperial Tarraco, s/n. Tarragona).

Seminari i grup d'estudis en Antropologia de la Medicina, de creació recent. Arxiu de documentació, (en formació).

- Centre d’Estudis Socialistes (Grup de Salut Pública). Vid: Butlletins.

- Catedra-Museo de Historia de la Medicina

Facultat de Medicina, Universitat de Valencia.

Dirigit pel Prof. Lopez Piñero disposa d'una biblioteca importantissima especialitzada i d'abundoses revistes en els camps sanitaris, historics i que facin referencia a temes de ciencies socials. Servei de fotocopies i microfilmació. (Vg. Centro de Documentación e informática biomédica).

\section{- Fundació-Museu d'Historia de la Medicina Catalana.}

Pje. Mercader 11, Barcelona.

Dirigit pel Prof. Cid es un centre en curs d'instal.lació. Biblioteca especialitzada (en formació). Actualment pendent d'inauguració al públic.

\subsection{Repertoris bibliogràfics:}

ASSOCIACIÓ GENERAL DE METGES DE LLENGUA CATALANA.

1918 Bibliografia Medical de Catalunya.

Barcelona, Impremta Elzeviriana.

Recopilació exhaustiva de bibliografia mèdica publicada a Catalunya des de l'Edat Mitjana.

GOL, Jordi et alii.

1976 La sanitat als Països Catalans.

Barcelona, Edicions 62.

779 referències sobre el tema sanitari a casa nostra.

GONZALEZ PAGE, Mercedes.

1976 Una bibliografia, comentada, sobre la planificació i reforma sanitària; pp: 455-485, en: Jesús de Miguel (comp) Planificación y Reforma sanitarias. Madrid, C.I. Sociológicas.

415 referències sobre el tema a l'Estat Espanyol. Amb l'anterior són les dues bibliografies més completes fins al 1975. 
GRANJEL, L. Y SANTANDER, Mercedes

1957 Bibliografía española de Historia de la Medicina.

Salamanca, Anaya.

Conté 1521 referències.

GRANJEL, L

1966 Bibliografia histórica de la Medicina Española. (2000-4000)

Salamanca, Universidad de Salamanca.

Conté quasi dues mil referències heterogènies.

PUJADAS, J.J., COMELLES, J.M. y PRAT, J.

1980 Una bibliografia comentada sobre Antropologia Mèdica, pp: 323. 353. A: Michael Kenny y Jesús de Miguel (comps) La Antropologia Médica en España. Barcelona, Anagrama.

Unes cinc-centes referències sobre Antropologia de la Medicina, encara que amb materials força heterogenis. Bastant completa fins al 1980, però no del tot. Próxima reedició ampliada a Arxiu d'Etnografia de Catalunya.

\subsection{Publicacions periòdiques.}

\subsubsection{Revistes de Ciències Socials.}

En aquests moments no existeix, a Espanya, cap publicació especificament reservada a investigacions en Sociologia i Antropologia de la Medicina. Podem destacar algunes publicacions que, amb certa regularitat, proposen articles en aquest camp; no podem, però, fer-ne una anàlisi com en el cas de la bibliografia peròdica estrangera.

- Revista de Dialectología y Tradiciones Populares. (C.S.I.C.) Ma drid.

Conté un nucli important d'articles clàssics en el folklore mèdic.

- Ethnica (C.S.I.C.) (Barcelona) Antropologia.

Quaderns de I'I.C.A. (Barcelona): Antropologia.

- Comentaris d'Antropologia Cultural (Barcelona): Antropologia.

Arxiu d'Etnografia de Catalunya. Antropologia.

Revista Española de Investigaciones Sociológicas, (abans) Revis. ta de la Opinión Pública. (C.S.I.C.) (Madrid): Sociologia.

- Papers. Revista de Sociologia (Barcelona).

La col.lecció sencera conté un fons important sobre Sociologia de la Medicina.

Revista de Higiene y Sanidad Pública. (Dirección General de Sanidad. Madrid).

Revista molt important pel seu fons.

- Temas de Antropologia aragonesa (Aragó, I.A.A.)

\subsubsection{Revistes de distribució a metges.}

- Jano (Barcelona)

- El Médico (Madrid)

- Pulso Peródico

- Consulta Semanal

- Doctor: Política profesional:

Revistes de distribució mèdica, que subministren, en base a informes més o menys exhaustius, una informació força ampla sobre el sector sanitari. Algunes destinen un cert espai al que podriem dir "ciències socials i salut". En qualsevol cas, permeten un se guiment setmanal i gairebé diari de la problemèticaelen un secasa nostra. La seva consulta és una font inapreciable de dades generals sobre el camp sanitari a Espanya.

\subsubsection{Butlletins.}

Algunes entitats, en els darrers temps, han començat a publicar butlletins interns que subministren informació general sobre Ciències socials $i$ salut.

\section{Salud y sociedad.}

Butlleti semestral del Grup de Sociologia de la Medicina. Informació general, notícies, anuncis, recensions, bibliografia.

- Butlletí d'informació sanitària.

Publicat pel grup de Salut Pública del Centre d'Estudis Socialis. tes (Rosselló 229, 3er. $1^{\text {a }}$ Barcelona-8). Conté informació general, notícies i comentaris, aixi com avisos $\mathrm{i}$ informacions sobre actes de tota mena relacionats amb aquesta materia.

\section{- Entaban}

Publicació del grup del mateix nom. (Apartat de Correus, 688. Saragossa). Presenta articles i recensions relatius al camp ut pública, I'Antropologia de la Medicina i la Socic dicina. 